\title{
FORUM
}

\section{Die Ukraine nach der Parlamentswahl 2007: neue Aussichten für die Integration?}

\author{
Manfred Grund*
}

Die orange Revolution des Jahres 2004 weckte bei ihren Anhängern und nicht wenigen Beobachtern auch im westlichen Ausland große Hoffnungen auf eine demokratische Erneuerung und eine schnelle Integration in die euro-atlantischen Strukturen. Was folgte, löste vielfach Ernüchterung aus: eine politische Dauerkrise mit drei nationalen Wahlen und vier Regierungswechseln in weniger als drei Jahren, deren vorläufigen Schlusspunkt der Urnengang vom 30. September 2007 darstellte.

Doch auch wenn sich allzu optimistische Einschätzungen nicht bestätigten, ist eine pessimistische Sicht ebenso fehl am Platze. Vor Stereotypen über die politischen Kräfte sollte man sich ebenfalls hüten. Schlichte Antagonismen bestimmen die Entwicklung allenfalls vordergründig. Der tatsächlichen Situation können nur nuancierte Urteile gerecht werden. Dies gilt nicht nur für die Beurteilung der orangen Revolution und ihrer Folgen, sondern auch der sich daraus ergebenden Aussichten für die Zukunft der Ukraine.

Dieser Befund schließt die Aussichten für eine weitere Annäherung an die Europäische Union ein. Da eine explizite Beitrittsperspektive vorerst nicht besteht, lassen sich die Chancen für eine Integration nur vor dem Hintergrund des Transformationsprozesses und der Konsolidierung der Demokratisierung in der Ukraine einschätzen. Erforderlich ist auch in dieser Hinsicht eine realistische Sicht, die Probleme beleuchtet, ohne die Fortschritte auszusparen.

\section{Eine politische Dauerkrise}

Zumindest vordergründig bestimmt das Bild einer politischen Dauerkrise die Entwicklung der Ukraine seit der orangen Revolution. Bruchlinien zeigten sich schon bald auch innerhalb des orangen Lagers, das in den Wahlen 2004/05 ebenso wie bei der Parlamentswahl von 2007 weniger durch eine gemeinsame Agenda zusammengehalten wurde als vielmehr durch eine gemeinsame Abwehrhaltung gegen einen sonst überlegenen Janukowytsch. Die nicht selten sozialpopulistischen Forderungen Tymoschenkos ließen sich jedoch mit den marktwirtschaftlichen Reformvorstellungen Juschtschenkos nur schwer vereinbaren. Zusammen mit Kompetenzstreitigkeiten mit der machtbewussten Tymoschenko war dies die tiefere Ursache für das Auseinanderbrechen der orangen Koalition weniger als ein Jahr nach der Wahl Juschtschenkos zum Präsidenten.

Ähnlich gelagerte Probleme drohen auch eine Neuauflage der orangen Koalition zu überschatten. Der Präsident hatte mit seinem Befreiungsschlag gegenüber Janukowytsch zwar vorerst Erfolg, doch droht ihm nun eine Marginalisierung durch eine allzu sehr dominierende Tymoschenko, die sich bereits auf eine Präsidentschaftskandidatur für 2009 vorbereiten dürfte. Die Mehrheitsverhältnisse sind jedoch so knapp und die Parteiloyalitäten der Abgeordneten so wenig ausgeprägt, dass sich ohne Einbeziehung des Parteienblocks des

* Manfred Grund, MdB, Parlamentarischer Geschäftsführer der CDU/CSU-Fraktion und Mitglied im Auswärtigen Ausschuss. 
früheren Parlamentspräsidenten Litvin schwerlich eine regierungsfähige Koalition der , orangen“ Kräfte bilden lässt. In der Opposition wartet die Partei der Regionen (PdR) auf ihre Chance. Ein Zugewinn an politischer Stabilität wird wohl kaum das Resultat der Wahlen allein sein.

Zur politischen Dauerkrise trat nach der orangen Revolution noch ein wirtschaftlicher Rückschlag. Preiserhöhungen für russische Energielieferungen waren dafür nur zum Teil verantwortlich. Willkürlich erscheinende Änderungen von Zoll- und Steuervorschriften, die Aufhebung der Sonderwirtschaftszonen und nicht zuletzt auch eine Revision früherer Privatisierungen richteten sich unter anderem gegen ukrainische Oligarchen, verunsicherten aber auch viele Investoren. Im Ergebnis brach das Wirtschaftswachstum der Ukraine 2005 zunächst drastisch ein.

Schließlich kam und kommt noch eine Verfassungs- und Staatskrise hinzu. Die Verfassungsreform, die Juschtschenko den Weg ins Präsidentenamt ebnete, stärkte die Rechte von Parlament und Regierung gegenüber dem zuvor herrschenden Präsidialregime, ließ aber eine Reihe wichtiger Entscheidungskompetenzen wie beispielsweise die Besetzung von Schlüsselressorts offen. Die Folge war eine Flut von Verfassungsklagen und eine seither latente Verfassungskrise. Sie wurde durch den Autoritätsverlust aller zentralen Verfassungsorgane infolge des Machtkampfs zwischen Premier Janukowytsch und Präsident Juschtschenko zusätzlich verschärft.

Parlamentsdebatten dienten mehr und mehr der Inszenierung von Gegensätzen unter oft chaotischen Umständen. Ganze Gruppen von Parlamentariern wechselten aufgrund augenscheinlich rein persönlicher Vorteile ins blaue Lager. Die verfassungsrechtlich umstrittene Parlamentsauflösung, die der Präsident anordnete, wurde zunächst ebenso wie andere seiner Verfügungen ignoriert. Dass er auf dem Höhepunkt der Krise mit der Staatsgewalt drohte, demonstrierte eher Machtverfall als Stärke. Auch das Verfassungsgericht fiel als Schlichter aus, teils aufgrund der Parteilichkeit seiner Mitglieder, teils aufgrund von Eingriffen des Präsidenten, der inmitten der Krise die Entlassung mehrerer Richter anordnete.

\section{Fortschritte im Transformationsprozess}

Die Krisen der vergangenen Jahre sind auch mit der Wahl vom September 2007 nicht ausgestanden. Sie sollten jedoch nicht über die Wandlungsprozesse hinwegtäuschen, die sich in der Ukraine inzwischen vollzogen haben. Fortschritte im Transformationsprozess demonstrierten nicht zuletzt die jüngsten Wahlen. Vorherige Befürchtungen vor Manipulationen erfüllten sich nicht. Die Berichterstattung und der Zugang zu Medien waren durchaus ausgewogen. Allerdings war die Gewähr dafür weniger eine Unabhängigkeit der Medien, als vielmehr der Umstand, dass die Zusammensetzung nahezu aller maßgeblichen Kontrollorgane inzwischen den politischen Kräfteverhältnissen entspricht. Die Ukraine ist in einem Entwicklungsstadium, in dem nahezu jede Aussage zu den Verhältnissen, gleichgültig ob positiv oder negativ, von einem relativierenden ,Aber' gefolgt werden muss.

Ähnliches gilt für die ökonomische Entwicklung. Das Wirtschaftswachstum hat sich seit 2005 wieder erholt. Reformen haben zu greifen begonnen. Die Höhe der ausländischen Direktinvestitionen - der beste Indikator für ihren Erfolg - hat sich seit 2004 vervierfacht. Die Europäische Union hat der Ukraine Ende 2005 den Status einer Marktwirtschaft zuerkannt. Doch die wirtschaftlichen Strukturen sind von einem echten Wettbewerb in vielen Bereichen tatsächlich noch weit entfernt. Korruption, schlecht funktionierende Verwaltungs- und Justizsysteme, die Verflechtung politischer und wirtschaftlicher Macht sowie Überregulierung bleiben grundlegende Probleme. 
Das Parteiensystem hat sich mit den beiden großen Fraktionen der PdR und dem Block Julija Tymoschenko (BJUT) zunehmend stabilisiert. Relativer Verlierer dieser Entwicklung ist mit dem Parteienbündnis Präsident Juschtschenkos jedoch die politische Kraft, die sich bislang am konsequentesten für eine Integration in die euro-atlantischen Strukturen eingesetzt hat. Neben politischen Fehlern hat ihn nicht zuletzt die zu den Herrschaftskompromissen nach seiner Amtsübernahme zählende Entscheidung, den Wahlfälschern von 2004 Amnestie zu gewähren, in seiner ursprünglichen Anhängerschaft nachhaltig diskreditiert. Dass sich sein Parteienbündnis entgegen früherer Prognosen behaupten konnte, war nicht zuletzt der Popularität von Spitzenkandidat Luzenko geschuldet. Als heteronomstes unter den drei großen politischen Blöcken wird es künftig einer ständigen Zerreißprobe zwischen den beiden größeren Kräften ausgesetzt sein.

Langfristig bedeutsamer dürfte jedoch sein, dass sich die Gegensätze zwischen den beiden großen Kräften des BJUT und der PdR abschwächen, auch wenn die Wahlergebnisse insgesamt nach wie vor die regionalen Unterschiede widerspiegeln. So hat es der BJUT verstanden, auch die Interessen von Bevölkerung und Geschäftsleuten der Ostukraine einzubinden, und entwickelt sich nach seinen zunehmenden Wahlerfolgen dort zur ersten gesamtukrainischen Partei. Janukowytschs Regierungsübernahme hat Desintegrationstendenzen in den östlichen und südlichen Landesteilen entgegengewirkt, zugleich aber verhindert, dass die PdR die regionalen Unterschiede ausspielen konnte. Zugleich aber vermochte sie ihre Verluste in Ost und Süd im Westen zu kompensieren.

Aber auch die politischen Unterschiede verlieren an Schärfe. Die Wahlprogramme der großen Parteien waren über weite Strecken austauschbar. Während die PdR sich zunehmend als gesamtukrainische Kraft zu profilieren suchte, kann die Beantragung eines Beobachterstatus in der Europäischen Volkspartei seitens des BJUT als Abrücken von sozialpopulistischen Forderungen gedeutet werden. Ohnehin wurzelten die Machtkämpfe der vergangenen Jahre oft mehr in den Rivalitäten der Protagonisten als in programmatischen Unterschieden; und sie wurden durch Kompromisslosigkeit bei der Berücksichtigung der Interessen der jeweiligen Opposition zusätzlich verschärft. Stellungnahmen Tymoschenkos und Juschtschenkos nach der Wahl scheinen aber auch in dieser Hinsicht ein Umdenken anzukündigen.

Stereotypen, nach denen ein an Russland angelehntes ,blaues‘ einem west- und reformorientierten orangen Lager gegenübersteht, sind endgültig überholt. Sie waren zu keinem Zeitpunkt hilfreich, weil so tief greifende Projekte wie die demokratische Transformation des Landes oder die EU-Integration dauerhaft nicht nur von einer Hälfte der politischen Kräfte getragen werden kann. Die wichtigste Voraussetzung für die Etablierung einer funktionierenden Demokratie besteht nicht aus dem Sieg vermeintlicher Demokraten über vermeintliche Nichtdemokraten, sondern aus der Entwicklung eines Wechselspiels von Regierung und Opposition. Daher ist das Comeback von Janukowytsch mit seiner PdR nach der orangen Revolution nur zu begrüßen.

Graustufen markieren die Unterschiede zwischen den maßgeblichen politischen Kräften. Janukowytsch zeigt sich inzwischen als geläuterter Machtpolitiker, der sich den neuen Spielregeln und Realitäten anzupassen vermochte. Dies gilt auch für Außenpolitik und Westintegration. Differenzen bestehen in der Frage einer NATO-Mitgliedschaft, die von der PdR in Übereinstimmung mit einer deutlichen Mehrheit in der Bevölkerung abgelehnt wird. Doch auch die PdR hat sich das Ziel eines EU-Beitritts zu eigen gemacht. Dass sie dabei einen pragmatischen Ansatz verfolgt, stellt eine Abweichung in der Akzentsetzung, nicht aber im Grundsatz dar. Darin spiegelt sich ein Wandel der Interessenlage, den die orange Revolution für alle politischen Lager bewirkt hat. 
Unabhängig von allen politischen Präferenzen sind dafür vor allem zwei Motive ausschlaggebend: erstens die Veränderung im Verhältnis zu Russland und zweitens wirtschaftliche Eigeninteressen. Mit der Entwicklung des politischen Pluralismus in der Ukraine gingen Irritationen und Entfremdungsprozesse zu Moskau nahezu zwangsläufig einher. Eine Entflechtung von Wirtschaftsstrukturen, insbesondere in der Rüstungsindustrie vollzieht sich bereits. Abhängigkeiten bestehen zwar fort. Sie ermöglichen es Russland, den Prozess der Westintegration zu erschweren, vielleicht auch zu verlangsamen, aber es muss sich dafür Mittel bedienen, die eine demokratische Ukraine in ihrem Kurs nur bestärken würden. Aus demselben Grund wie aufgrund der Bevölkerungsstruktur wird jede ukrainische Regierung eine pragmatische Politik gegenüber Moskau betreiben, auch wenn zwischen Tymoschenko, die vom Erfordernis einer Eindämmung Russlands spricht, und Janukowytsch ebenfalls unterschiedliche Akzentsetzungen herrschen. Zugleich aber ist allen Protagonisten bewusst, dass die Ukraine auch gegenüber Moskau in stärkerem Maße als bisher auf die Unterstützung des Westens angewiesen sein wird.

Wirtschaftliche Eigeninteressen verbinden sich mit einer EU-Integration insbesondere auch für ukrainische Oligarchen, die auf die ukrainischen Parteien und nicht zuletzt auf die PdR nach wie vor einen kaum zu überschätzenden Einfluss haben. Dies betrifft nicht nur Absatzmärkte, von denen vor allem die ostukrainische Industrie profitieren würde, oder ausländisches Kapital, auf das die ukrainische Industrie zu ihrer Modernisierung angewiesen ist, sondern auch die Sicherung von Besitzständen. War Regierungsnähe unter Kutschma ein Faktor wirtschaftlicher Vorteile, ist sie mit der Aussicht auf mögliche Machtwechsel zum Risikofaktor geworden. Dass die Privatisierung des Stahlwerks Kriworoschstal, das ein Konsortium um Achmetov und Pintschuk zuvor erworben hatte, nach der orangen Revolution aufgehoben wurde, demonstrierte nur allzu deutlich, dass schnell erworbene Vermögen ebenso schnell wieder verloren gehen können, wenn sie nicht durch rechtsstaatliche Garantien abgesichert werden. Eine EU-Integration aber verspräche auch diese entscheidend zu stärken.

\section{Welche Integrationsperspektive für die Ukraine?}

Eine zunehmende Westorientierung liegt in der inneren Logik der Entwicklung, die die Ukraine seit der orangen Revolution genommen hat. Die Entscheidung für eine EU-Integration dürfte im Prinzip unumkehrbar sein. Bereits in ihrer gemeinsamen Strategie gegenüber der Ukraine hat die Europäische Union die ,European Aspirations ' Kiews anerkannt und die Möglichkeit einer künftigen Mitgliedschaft seither stets als offen behandelt. Zugleich aber wurde die Nachbarschaftspolitik explizit als Alternative zum Beitritt entwickelt. Ob eine Vertiefung der Nachbarschaftspolitik das Fehlen des Reformanreizes der Beitrittsperspektive ersetzen kann, muss sich noch zeigen.

Die Verhandlungen über ein erweitertes Nachfolgeabkommen des auslaufenden Partnerschafts- und Kooperationsabkommens wurden auch während der ukrainischen Krise weitgehend unbeeinträchtigt fortgesetzt. Dem Begriff nach könnte im Ergebnis ein Assoziationsabkommen stehen, das für europäische Staaten bislang stets den Beginn eines Beitrittsprozesses markierte. Herzstück der neuen Vereinbarung soll die Einrichtung einer gemeinsamen Freihandelszone werden, sobald die Ukraine der WTO beigetreten ist. Das Bestreben, dieses Ziel nicht zu gefährden, dürfte zumindest kurzfristig einen moderierenden Einfluss auf die Auseinandersetzungen zwischen den politischen Kräften in der Ukraine haben. Dies mag dokumentieren, welche Bedeutung die Integration inzwischen parteiübergreifend gewonnen hat. 
Von Seiten der Europäischen Union ist jedoch zunächst keine explizite Beitrittsperspektive zu erwarten. Hinsichtlich des Transformationsprozesses in der Ukraine und der damit noch verbundenen Unsicherheiten wäre ein solches Zeichen aber auch verfrüht. Eine Voraussetzung wäre, dass die Ukraine ihre politische Dauerkrise überwindet und zu einer konsequenten Reformpolitik findet. In der Zwischenzeit bedürfte es vielmehr auch von Seiten der Europäischen Union einer pragmatischen Politik der schrittweisen Vertiefung und Ausweitung der Integration. Ein Grundproblem der Nachbarschaftspolitik liegt nicht darin, dass sie keine Beitrittsperspektive gewährt, sondern dass sie sich nicht von der Logik der bisherigen Erweiterungsprozesse löst, nach der nur eine formale Beitrittsperspektive die spätere Mitgliedschaft zu versprechen scheint.

Einer Ukraine, die die Beitrittskriterien erfüllt, und die den acquis bereits im Zuge ihrer EU-Annäherung weitgehend übernommen hätte, wird die Mitgliedschaft kaum verwehrt bleiben können. So gesehen, besteht für die Ukraine bereits eine Perspektive zur Perspektive oder eben eine implizite Beitrittsperspektive. Diese Feststellung sollte - und wird auch zunehmend - den Kern der Botschaft bilden, die die Europäische Union der ukrainischen Politik vermittelt. Die Antwort auf die Beitrittswünsche der Ukraine ist nicht von vornherein in Brüssel zu finden, sie muss zuerst im eigenen Land gegeben werden, mit dem Fortschritt der Reformen.

\section{Europäische Beschäftigungspolitik}

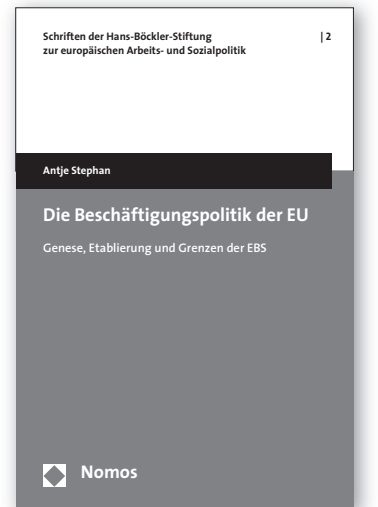

Die Beschäftigungspolitik der EU

Genese, Etablierung und Grenzen der EBS

Von Antje Stephan

2008, ca. 350 S., brosch., ca. 64,- $€$,

ISBN 978-3-8329-3235-O

(Schriften der Hans-Böckler-Stiftung zur europäischen

Arbeits- und Sozialpolitik, Bd. 2)

Erscheint April 2008

Europäische Beschäftigungsstrategie, Offene Methode der Koordinierung und Lissabon-Strategie - so vielfältig wie die Ansätze der EU sind, zu mehr Beschäftigung zu gelangen, so vielfältig sind auch die Gründe, warum dies bislang nur unzureichend gelungen ist. Diese Studie liefert erstmals eine multidisziplinäre Erklärung für die bisher magere Erfolgsbilanz.

Bitte bestellen Sie bei Ihrer Buchhandlung oder bei Nomos | Telefon 07221/2104-37 | Fax -43 www.nomos.de | sabine.horn@nomos.de

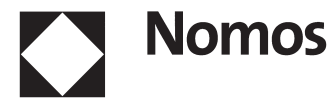

